\title{
Flow around a confined cylinder: LES and PIV study
}

\author{
Egor Palkin ${ }^{1,2, *}$, Mikhail Shestakov ${ }^{1,2}$, Rustam Mullyadzhanov ${ }^{1,2}$, Dmitry Markovich $^{1,2}$, and \\ Kemal Hanjalić ${ }^{2,3}$ \\ ${ }^{1}$ Institute of Thermophysics SB RAS, 1 Lavrentyev Ave., 630090 Novosibirsk, Russia \\ ${ }^{2}$ Novosibirsk State University,2 Pirogov Str., 630090 Novosibirsk, Russia \\ ${ }^{3}$ Delft University of Technology, Bld. 58, Van der Maasweg 9, 2629 HZ Delft, The Netherlands
}

\begin{abstract}
We study the flow over a cylinder placed between two parallel rigid walls using Large-eddy simulations and Particle Image Velocimetry. The Reynolds number based on the inflow velocity and diameter of the cylinder is 3750 corresponding to the subcritical regime with laminar separation. Three-dimensional visualization shows the presence of the horseshoe vortex system prior to the cylinder. The comparison of timeaveraged velocity fields and fluctuations shows good agreement between simulations and experiments. Spectral analysis suggests the presence of low-frequency modulations of the recirculating bubble.
\end{abstract}

\section{Introduction}

Flows over obstacles in a duct are common in many engineering applications such as cooling systems, bridge piers, heat exchangers, building sections, junctions in wing-body and turbine blade-rotor systems, among others. In such configurations a horseshoe vortex system appears prior to a bluff body increasing the local shear stress and heat transfer [1] while the flow is characterized by periodic shedding of large-scale vortices behind the body that form the Kármán vortex street. Low-frequency modulations of the recirculating zone are detected for various configurations such as a cylinder [2, 3], disk and sphere [4], prism [5], bullet [6], among others. The period is typically $10 \div 100$ times lower compared to the main vortex shedding frequency. In the present work we consider a flow over confined cylinder in a narrow rectangular duct to investigate the effect of walls on the dynamics of the recirculation bubble.

\section{Computational and experimental details}

We study a water flow over a circular cylinder which is fixed perpendicular to a pair of side walls at the Reynolds number $R e=3750$ based on the bulk inflow velocity $U_{b}$ and cylinder diameter $D$. The inflow velocity distribution represents a steady laminar parabolic profile. The distance between narrow parallel walls is $H=0.4 \mathrm{D}$. The case is studied using numerical simulations and experiments described below.

*Corresponding author: palkinev89@gmail.com 

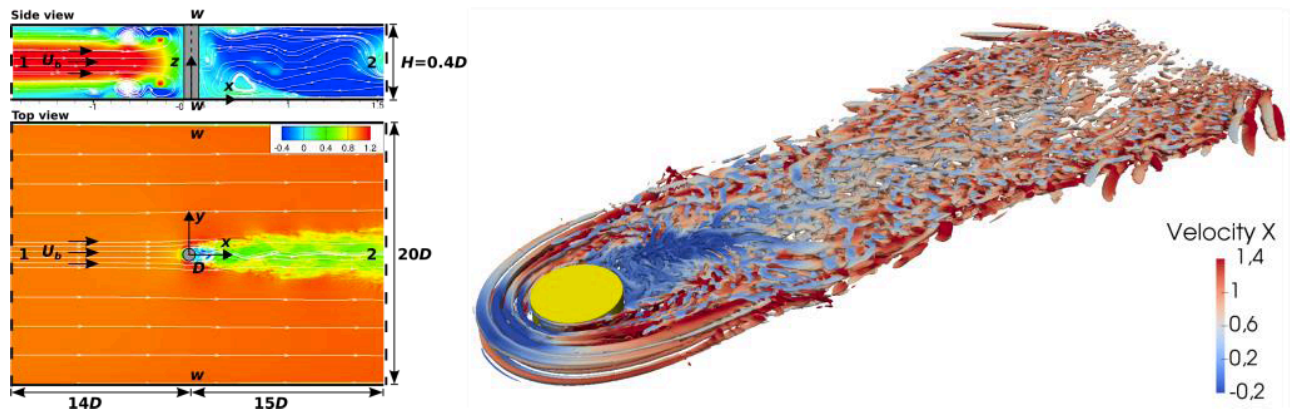

Fig. 1. Left: Schematic view of computational domain with instantaneous streamwise velocity field and streamlines. The boundary conditions correspond to the following marks: ' 1 ' - inflow, ' 2 ' outflow, ' $w$ ' - no-slip (walls). Right: isosurface of Q-criterion to visualize vortical structures colored with the streamwise velocity.

We perform Large-eddy simulations (LES) using the unstructured finite-volume computational code T-FlowS. The filtered Navier-Stokes and continuity equations for incompressible fluid are closed with the dynamic Smagorinsky subgrid-scale model. The spatial discretization is performed with the second-order central-difference scheme, whereas for the time-marching we use a fully-implicit three-level scheme. The velocity and pressure are coupled with the SIMPLE algorithm. The computational domain shown in Fig. 1 represents a box with a size $x \times y \times z=29 \mathrm{D} \times 20 D \times H$, where $x, y, z$ stand for the streamwise, spanwise and wall-normal directions. The computations were performed on two meshes with $8.7 \times 10^{6}$ and $16.6 \times 10^{6}$ cells, respectively, with no significant differences in the results. Both meshes satisfy wall-resolved LES criteria. In particular, even the 'coarse' mesh corresponds to high resolution since the first cell near the cylinder did not exceed the following limits: $\Delta r^{+}<1,(R \Delta \varphi)^{+}<8$ and $\Delta z^{+}<4$, where '+' denotes the wall units and $R=D / 2$. The total computational time was around $10^{3} D / U_{b}$ with a nondimensional timestep $2.5 \times 10^{-3}$.

The experiments were performed in a slot channel with the length and width of $38 \mathrm{D}$ and $20 D$, respectively, where $D=10 \mathrm{~mm}$. In order to provide steady velocity distribution close to parabolic at the inflow, the flow passed through a set of two honeycombs. Velocity fields were measured using Particle Image Velocimetry (PIV) technique. The system consists of a digital PCO camera $(1024 \times 1280$ pix, $500 \mathrm{~Hz}$ max. frame rate $)$ and dual cavity Nd:YAG laser $(1000 \mathrm{~Hz}$ max. rate, $10 \mathrm{~mJ}$ max. pulse energy). The camera was located perpendicular to the main channel. The thickness of the laser sheet was equal to $0.7 \mathrm{~mm}$. PIV measurements were performed in a $2 D \times 2 \mathrm{D}$ region behing the cylinder. The averaged characteristics were calculated using 1000 instantaneous velocity fields. The spatial resolution was estimated to be $0.3 \mathrm{~mm}$.

\section{Results}

The flow regime corresponds to the subcritical one at this relatively low Reynolds number with the separation of the laminar boundary layer and subsequent turbulization of the shear layer. A highly three-dimensional flow appears in the near wake region within the recirculating bubble due to the bounding narrow walls. The hourseshoe vortices decay while interacting with the shear-layer turbulence (Fig. 1). Further downstream the wake becomes fully developed. Figures 2 and 3 demonstrate the time-averaged axial velocity for LES and PIV results together with the comparison at certain axial locations. The length of the recirculating zone is slightly shorter for LES $(\approx 1.3 D)$, while overall results are in a satisfactory agreement with PIV measurements. The influence of horseshoe vortices are 
clearly visible on the mean streamwise velocity around $|y| / D \approx 0.7$ showing a pronounced drop. PIV results capture this effect on $x / D=1.2$ profile of mean $U / U_{b}$.
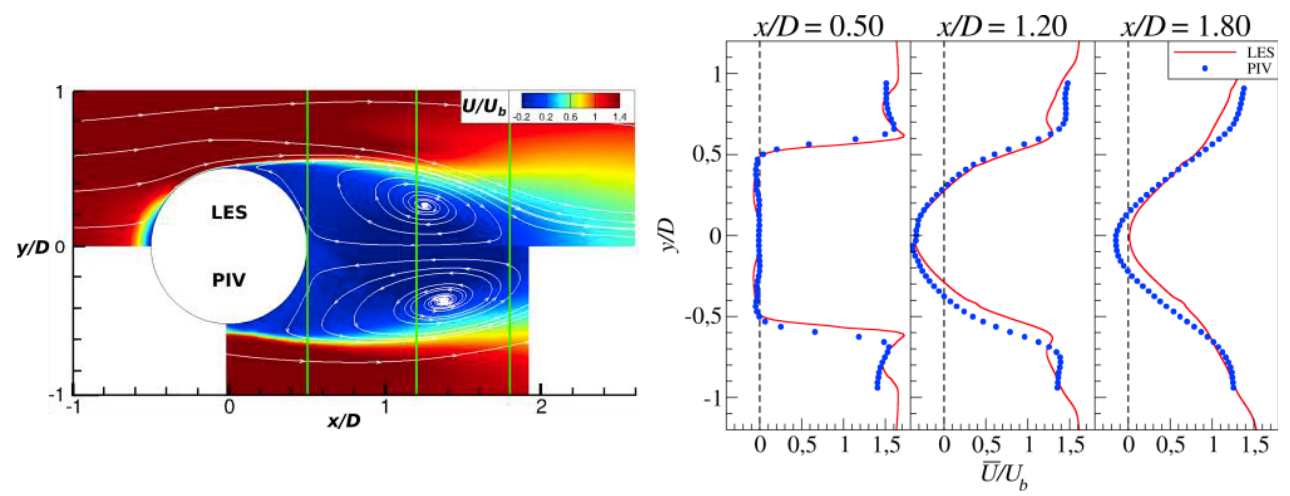

Fig. 2. Left: The field of the time-averaged axial velocity and streamlines from LES (top half) and PIV (bottom). Right: The profiles of the time-averaged axial velocity at three axial locations shown on the left by green vertical lines.
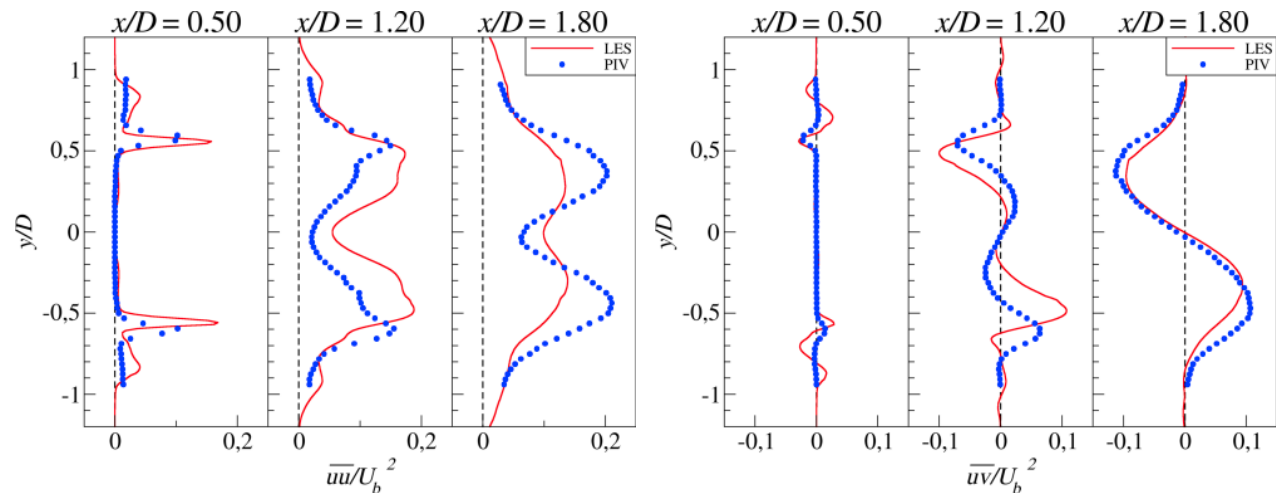

Fig. 3. Profiles of the normal streamwise (left) and shear (right) stresses at the same axial locations as in Fig. 2.

Further we perform spectral analysis of velocity fluctuations to detect characteristic frequencies of the flow, Fig. 4. The comparison of the time-averaged normal spanwise Reynolds stress components shows excellent agreement between LES and PIV. The dominant peak is around $S t=f D / U_{b} \approx 0.23$ corresponding to the vortex shedding frequency. The component mean $w w$ was unavailable in PIV and the comparison could not be performed. LES data reveals some low-frequency fluctuations with the peaks around $S t \approx$ 0.04 . Future work will be devoted to identifying the hydrodynamics behind these lowfrequency oscillations. 

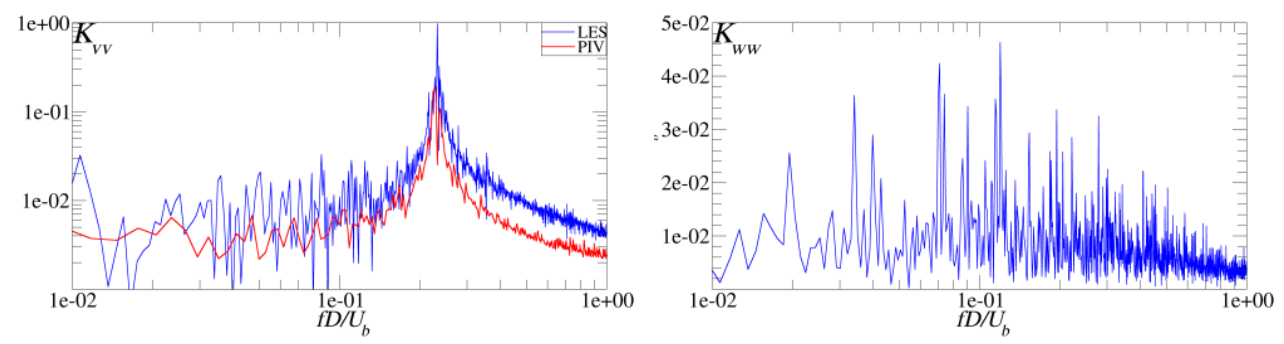

Fig. 4. Power spectra of velocity fluctuations close to the cylinder at the centreline, $(x / D, y / D, z / H)=$ $(0.7,0.0,0.5)$. Left: spanwise component from LES and PIV. Right: wall-normal fluctuations.

\section{Discussion}

We performed LES and PIV of the flow over a confined cylinder in a narrow duct at $\mathrm{Re}=$ 3750. Spectral analysis suggests the presence of low-frequency modulations of the recirculating bubble. This will be the topic for the future study. Another issue is the effect of bounding walls on the developed wake. Our observations $[7,8]$ in confined jets revealed the existence of streamwise meandering vortices in the flow influencing the heat transfer across the channel. It is expected that a similar phenomenon should be present in a confined wake flow.

This work is funded by the Russian Science Foundation grant No. 14-19-01685. The computational resources are provided by Siberian Supercomputer Center SB RAS and Supercomputing Center of the Novosibirsk State University.

\section{References}

1. R. Simpson, Ann. Rev. Fluid Mech. 33, 415 (2001)

2. O. Lehmkuhl, I. Rodriguez, R. Borrell, A. Oliva, Phys. Fluids 25, 085109 (2013)

3. E. Palkin, R. Mullyadzhanov, M. Hadziabdic, K. Hanjalic, Flow Turbul. Combust. 97, 1017 (2016)

4. E. Berger, D. Scholz, M. Schumm, J. Fluid Struct. 4, 231 (1990)

5. J. Miau, J. Wang, J. Chou, C. Wei, J. Fluid Struct. 13, 339 (1999)

6. V. Gentile, F. F. J. Schrijer, B. W. Van Oudheusden, F. Scarano, Phys. Fluids 28, 065102 (2016)

7. R. Mullyadzhanov, B. Ilyushin, K. Hanjalic, Int. J. Heat Fluid Flow 56, 284 (2015)

8. M. Shestakov, R. Mullyadzhanov, M. Tokarev, D. Markovich, J. Eng. Thermophys. 25, 159 (2016) 